\title{
A Transformation Method for Delta Partial Difference Equations on Discrete Time Scale
}

\author{
Syed Sabyel Haider $\mathbb{D D}^{1}$ Mujeeb Ur Rehman, ${ }^{1}$ and Thabet Abdeljawad ${ }^{20,3,4}$ \\ ${ }^{1}$ School of Natural Sciences, National University of Sciences and Technology, H-12, Islamabad, Pakistan \\ ${ }^{2}$ Department of Mathematics and General Sciences, Prince Sultan University, P.O. Box 66833, Riyadh 11586, Saudi Arabia \\ ${ }^{3}$ Department of Medical Research, China Medical University, Taichung 40402, Taiwan \\ ${ }^{4}$ Department of Computer Science and Information Engineering, Asia University, Taichung, Taiwan
}

Correspondence should be addressed to Thabet Abdeljawad; tabdeljawad@psu.edu.sa

Received 3 May 2020; Revised 28 May 2020; Accepted 18 June 2020; Published 10 July 2020

Guest Editor: Baogui Xin

Copyright ( 2020 Syed Sabyel Haider et al. This is an open access article distributed under the Creative Commons Attribution License, which permits unrestricted use, distribution, and reproduction in any medium, provided the original work is properly cited.

\begin{abstract}
The aim of this study is to develop a transform method for discrete calculus. We define the double Laplace transforms in a discrete setting and discuss its existence and uniqueness with some of its important properties. The delta double Laplace transforms have been presented for integer and noninteger order partial differences. For illustration, the delta double Laplace transforms are applied to solve partial difference equation.
\end{abstract}

\section{Introduction}

The origin of calculus of finite differences is found from Brook Taylor (1717), rather it was Jacob Stirling, who found the theory (1730) and introduce the delta $\Delta$ symbol for the difference, which is in common use nowadays. The development on calculus of finite differences in the beginning of the nineteenth century by Lacroix and remarkable work of George Boole, Narlund, and Steffensen appeared later in the nineteenth century. Jordan discussed calculus of finite differences with the classical approach in [1]. In modern era, the focus of mathematician is to correlate the continuous and the discrete, to shape in comprehensive unified mathematics, and to eliminate ambiguity. The calculus of finite differences is applicable to both continuous and discrete functions. For difference equations, Bohner and Peterson treat the dynamic equations on time scales in [2] and get surprisingly different results from continuous counterpart. Some results can be found in [3-19] which has helped to construct the theory of discrete fractional calculus.

Coon and Bernstein [20-22] defined the double Laplace transforms (continuous) and investigated many properties. Debnath [23] modified the properties and use the double
Laplace transforms (continuous) to solve functional, integral, and partial differential equations. Dhunde and Waghmare [24] discussed convergence and absolute convergence of the double Laplace transforms (continuous) and, by application of double Laplace transforms, presented the solution of Volterra Integropartial differential equation. For applications of triple, quadruple, and $n$-dimensional Laplace transforms (continuous), we refer the readers to [25-27]. Goodrich and Peterson [10] developed discrete delta Laplace transforms analogous to Laplace transforms discussed by Bohner and Peterson [2] in the continuous case, to solve difference and summation equations with initial data by applying the delta Laplace transforms. The delta Laplace transforms is given for newly defined Hilfer difference operator [28] and substantial difference operator in [29]. Bohner et al. [30] generalized properties of the Laplace transforms to the delta Laplace transforms on arbitrary time scales and discussed translation theorems and transforms of periodic functions. Compatible discrete time Laplace transforms with Laplace transforms was introduced in [31]. Savoye [32] highlighted the importance of discrete time problems and relationship of $Z$ transforms to Laplace transforms on time scale. Fractional double Laplace 
transform was introduced in [33]; during derivation of Corollary 1, authors neglected the violation of semigroup property of Mittage-Leffler functions, and a counter example for semigroup property of Mittage-Leffler functions is given in [34]. The qualitative analysis of delay partial difference equations is considered as discrete analog of delay partial differential equations by Zhang and Zhou [35]. For solving partial difference equations Ozpinar and Belgacem introduced discrete homotopy perturbation Sumudu transform method in [36]. For solving partial differential equations, double Laplace transform was applied in [37, 38].

Here, we introduce the delta double Laplace transforms similar to the one presented by Bernstein [20] in such a way that properties and expressions bear a resemblance to that appearing in Debnath [23] for the continuous calculus. The double convolution product, we consider in this article, resemble with the convolution product defined for delta calculus in $[2,10]$, but it differs from the one defined by Atici in [8]. We consider the problem with constant coefficients in two independent variables and solve by applying the delta double Laplace transforms to partial difference equations with initial data.

This paper is divided into five sections. In Section 2, we shall present basic definitions and results from discrete calculus. Definition, existence, uniqueness, and series representation of the delta double Laplace transforms are given in Section 3. Some properties of the delta double Laplace transforms are proved in Section 4. In Section 5, we present the delta double Laplace transforms of partial differences.

\section{Preliminaries}

For convenience, this section comprises of some basic definitions and results from discrete calculus for later use in the following sections. The functions we consider usually are defined on the set $\mathbb{N}_{a}:=\{a, a+1, a+2, \ldots\}$ and $\mathbb{N}_{a}^{b}:=\{a, a+1, a+2, \ldots, b\}$, for fixed $a, b \in \mathbb{R}$.

The following concepts are discussed in $[10,16]$.

Falling function is defined for positive integer $n$ by

$$
x^{\underline{n}}=x(x-1)(x-2) \cdots(x-n+1) .
$$

The forward jump operator is defined for $x \in \mathbb{N}_{a}$ by $\sigma(x)=x+1$.

The set of regressive functions is defined for $x \in \mathbb{N}_{a}$ by $\mathscr{R}=\left\{p_{i}: 1+p_{i}(x) \neq 0\right\}$.

The circle plus sum of $p_{1}, p_{2} \in \mathscr{R}$ is given by $p_{1} \oplus p_{2}=p_{1}+p_{2}+p_{1} p_{2}$.

The additive inverse of $p_{1} \in \mathscr{R}$ is given by $\ominus p_{1}(x)=$ $-p_{1}(x) /\left(1+p_{1}(x)\right)$ for $x \in \mathbb{N}_{a}$.

Definition 1 (see [10]). Assume $p_{1} \in \mathscr{R}$ and $s \in \mathbb{N}_{a}$. Then, the delta exponential function is given by

$$
e_{p_{1}}(x, s)= \begin{cases}\prod_{t=s}^{x-1}\left[1+p_{1}(t)\right], & \text { if } x \in \mathbb{N}_{s}, \\ \prod_{t=x}^{s-1}\left[1+p_{1}(t)\right]^{-1}, & \text { if } x \in \mathbb{N}_{a}^{s-1}\end{cases}
$$

By the empty product convention, $\prod_{t=s}^{s-1}[h(t)]:=1$ for any function $h$.
Example 1. If $p_{1}(x)=c$ is a constant such that $c \in \mathscr{R}$ (that is $c \neq-1$ ), then the delta exponential function for a constant is given by

$$
e_{p_{1}}(x, s)=e_{c}(x, s)=[1+c]^{x-s}, \quad \text { for } x \in \mathbb{N}_{a} .
$$

For a particular choice of $s=a$, that is, the initial point of the domain of definition,

$$
e_{c}(x, a)=[1+c]^{x-a}, \quad \text { for } x \in \mathbb{N}_{a} .
$$

Definition 2 (see [10]). Assume $f: \mathbb{N}_{a} \longrightarrow \mathbb{R}$ and $b \leq c$ are in $\mathbb{N}_{a}$; then, the delta definite integral is defined by

$$
\int_{b}^{c} f(x) \Delta x=\sum_{x=b}^{c-1} f(x) .
$$

Note that the value of integral $\int_{b}^{c} f(x) \Delta x$, depending on the set $\{b, b+1, \ldots, c-1\}$. Also, by the empty sum convention,

$$
\sum_{x=b}^{b-k} f(x)=0, \quad \text { whenever } k \in \mathbb{N}_{1} .
$$

The delta indefinite integral is defined by

$$
\int_{b}^{\infty} f(x) \Delta x=\sum_{x=b}^{\infty} f(x) .
$$

Definition 3 (see [10]). Assume $f: \mathbb{N}_{a} \longrightarrow \mathbb{R}$. Then, the delta Laplace transform of $f$ based at $a$ is defined by

$$
\mathscr{L}_{x}\{f\}(p)=\int_{a}^{\infty} e_{\ominus p}(\sigma(x), a) f(x) \Delta x,
$$

for all complex numbers $p \neq-1$ such that this improper integral converges.

Note that throughout this article, we take the delta Laplace transform at the initial point $a$ of the set $\mathbb{N}_{a}$, unless stated otherwise.

The following concepts are also discussed in $[10,16]$.

Definition 4 (see [10]). A function $f$ is of exponential order $r_{1}>0$ if there exist a constant $A_{1}>0$ and the following inequality:

$$
|f(x)| \leq A_{1} r_{1}^{x}, \quad \text { holds for sufficiently large } x \in \mathbb{N}_{a} .
$$

If $f$ is of exponential order, then $\mathscr{L}_{x}\{f\}(p)$ converges absolutely for $|p+1|>r_{1}$, which ensures the existence of the Laplace transform. Even though the converse in not true, we restrict ourselves to only exponential order functions. For $f: \mathbb{N}_{a} \longrightarrow \mathbb{R}$, the following are useful expressions for the delta Laplace transform of $f$ based at $a$ :

$$
\begin{aligned}
\mathscr{L}_{x}\{f\}(p) & =\widetilde{F}(p)=\int_{0}^{\infty} \frac{f(a+j)}{(p+1)^{j+1}} \Delta j \\
& =\sum_{j=0}^{\infty} \frac{f(a+j)}{(p+1)^{j+1}},
\end{aligned}
$$

for all complex numbers $p \neq-1$ such that this infinite series converges. 
Example 2. If $c \neq-1$, then for $|p+1|>|c+1|$, we have

$$
\mathscr{L}_{x}\left\{e_{c}(x, a)\right\}(p)=\frac{1}{p-c} .
$$

Definition 5 (see [10]). Assume $f, g: \mathbb{N}_{a} \longrightarrow \mathbb{R}$. The convolution product is defined by

$$
(f * g)(x)=\sum_{r=a}^{x-1} f(r) g(x-\sigma(r)+a), \quad \text { for } x \in \mathbb{N}_{a} .
$$

Note that by the empty sum convention $(f * g)(a)=0$.

Lemma 1 (convolution theorem, see [10]). Assume $f, g: \mathbb{N}_{a} \longrightarrow \mathbb{R}$. If both $\mathscr{L}_{x} f(x)$ and $\mathscr{L}_{x} g(x)$ exist, then the delta Laplace transform of the convolution product is given by

$$
\mathscr{L}_{x}\{(f * g)(x)\}=\mathscr{L}_{x}\{f(x)\} \mathscr{L}_{x}\{g(x)\}=\mathscr{L}_{x}\{(g * f)(x)\} .
$$

Lemma 2 (see [10]). Assume two functions $v, w: \mathbb{N}_{a} \longrightarrow \mathbb{R}$. Let $b_{1}, b_{2} \in \mathbb{N}_{a}$ such that $b_{1}<b_{2}$, and we have the summation by parts formula:

$$
\int_{b_{1}}^{b_{2}} v(\sigma(t)) \Delta w(t) \Delta t=\left.v(t) w(t)\right|_{b_{1}} ^{b_{2}}-\int_{b_{1}}^{b_{2}} w(t) \Delta v(t) \Delta t .
$$

Definition 6. The generalized falling function is defined in term of gamma function by

$$
t^{\mu}=\frac{\Gamma(\sigma(t))}{\Gamma(\sigma(t)-\mu)}, \quad \text { for } t \in \mathbb{N}_{a}, \mu \in \mathbb{R},
$$

given that the expression in the above equation is justifiable. It is convenient to take $t_{-}^{\mu}=0$, whenever $t+1$ is natural number and $t-\mu+1$ is a zero or negative integer.

Definition 7. The discrete Taylor monomial based at $s=a$ is defined by

$$
h_{n}(x, a)=\frac{(x-a)^{\frac{n}{}}}{n !}, \quad \text { for } x \in \mathbb{N}_{a}
$$

and the $\mu^{\text {th }}$ order Taylor monomial is defined by

$$
h_{\mu}(x, a)=\frac{(x-a)^{\underline{\mu}}}{\Gamma(\mu+1)}, \quad \text { for } x \in \mathbb{N}_{a} .
$$

Lemma 3 (see [10]). Ie following hold for delta Laplace of Taylor monomial:

$$
\begin{aligned}
& \text { (i) } \mathscr{L}_{x}\left\{h_{n}(x, a)\right\}(p)=1 / p^{n+1}, \text { for }|p+1|>1, n \in \mathbb{N}_{0} \\
& \text { (ii) } \mathscr{L}_{x}\left\{(x-a)^{\underline{n}}\right\}(p)=n ! / p^{n+1}, \text { for }|p+1|>1, n \in \mathbb{N}_{0} \\
& \text { (iii) } \mathscr{L}_{x}\left\{(x-a)^{\mu}\right\}(p)=\left(\Gamma(\mu+1)(p+1)^{\mu}\right) / p^{\mu+1}, \quad \text { for } \\
& |p+1|>1, \mu \geq 0 .
\end{aligned}
$$

In the next definition, we consider only delta difference with increment 1 , and do not bother the different operators that we will not be using here. One can find the details of Definition 8 in $[1,39]$.

Definition 8. Assume $u: \mathbb{N}_{a} \times \mathbb{N}_{a} \longrightarrow \mathbb{R}$, a function of two independent variables. Then, the partial difference of $u(x, y)$ with respect to $x$, regarding $y$ as a constant is given by

$$
\Delta_{x}[u(x, y)]=u(x+1, y)-u(x, y) .
$$

The partial difference of $u(x, y)$ with respect to $y$, regarding $x$ as a constant, is given by

$$
\Delta_{y}[u(x, y)]=u(x, y+1)-u(x, y) .
$$

Partial difference equation is an equation containing partial differences.

Note that $\Delta_{x y}=\Delta_{y} \Delta_{x}=\Delta_{x} \Delta_{y}=\Delta_{y x}$. Followed by the rule for integer order difference operator $\Delta^{n}=\Delta \Delta^{n-1}$, we adopt the symbol for partial differences as follows: $\Delta_{x}^{n}=$ $\Delta_{x} \Delta_{x}^{n-1}$ and $\Delta_{y}^{m}=\Delta_{y} \Delta_{y}^{m-1}$.

\section{The Delta Double Laplace Transforms}

In this section, we give abstract definition of the delta double Laplace transform. For convenience, we simplify definition to series representation followed by Goodrich and Peterson [10] simplification of the delta Laplace transform. Also, condition for existence, uniqueness, and linearity of the delta double Laplace transform has been revealed.

Definition 9. Assume $f: \mathbb{N}_{a} \times \mathbb{N}_{a} \longrightarrow \mathbb{R}$. Then, the delta double Laplace transform of $f$ based at $(a, a)$ is the successive application of the delta Laplace transform on $x$ and $y$ in any order

$$
\begin{aligned}
\mathscr{L}_{2}[f(x, y)](p, q) & =\mathscr{L}_{x}\left[\mathscr{L}_{y}\{f(x, y) ; y \longrightarrow q\} ; x \longrightarrow p\right] \\
& =\mathscr{L}_{y}\left[\mathscr{L}_{x}\{f(x, y) ; x \longrightarrow p\} ; y \longrightarrow q\right] \\
& =\mathscr{L}_{y}[\widetilde{F}(p, y) ; y \longrightarrow q] \\
& =\widetilde{\widetilde{F}}(p, q),
\end{aligned}
$$

where $\mathscr{L}_{x}$ and $\mathscr{L}_{y}$ are the delta Laplace transforms (single) based at $a$ with respect to $x$ and $y$, respectively, and $\mathscr{L}_{2}$ is the delta double Laplace transform based at $(a, a)$. The delta double Laplace transform of a function $f(x, y)$ of two variables $x$ and $y$ is defined in $p-q$ plane provided the following double sum converges:

$$
\mathscr{L}_{2}\{f\}(p, q)=\int_{a}^{\infty} \int_{a}^{\infty} e_{\ominus p}(\sigma(x), a) e_{\ominus q}(\sigma(y), a) f(x, y) \Delta x \Delta y,
$$

for all complex numbers $p \neq-1$ and $q \neq-1$.

One can easily verify by using Lemma 4 that $\mathscr{L}_{x} \mathscr{L}_{y}=\mathscr{L}_{y} \mathscr{L}_{x}$. Later, in Theorem 2, we will prove that the double infinite series is absolutely convergent. It is well known that absolutely convergent series behave nicely and change in the order of summation $\sum_{k=0}^{\infty} \sum_{j=0}^{\infty}$ allowed. Therefore, we can operate in either way $\mathscr{L}_{x} \mathscr{L}_{y}=\mathscr{L}_{y} \mathscr{L}_{x}$. 
Lemma 4. Assume $f: \mathbb{N}_{a} \times \mathbb{N}_{a} \longrightarrow \mathbb{R}$. Then,

$$
\mathscr{L}_{2}[f(x, y)]=\sum_{k=0}^{\infty} \sum_{j=0}^{\infty} \frac{f(a+j, a+k)}{(p+1)^{j+1}(q+1)^{k+1}},
$$

for all complex numbers $p \neq-1$ and $q \neq-1$ such that the infinite series converges.

Proof. By using the definition of the delta double Laplace transform, consider the following:

$$
\mathscr{L}_{2}\{f\}(p, q)=\int_{a}^{\infty} \int_{a}^{\infty} e_{\ominus p}(\sigma(x), a) e_{\ominus q}(\sigma(y), a) f(x, y) \Delta x \Delta y .
$$

Now, by the definition of delta integral from discrete calculus, we obtain

$$
\begin{aligned}
& =\sum_{y=a}^{\infty} \sum_{x=a}^{\infty} e_{\ominus p}(\sigma(x), a) e_{\ominus q}(\sigma(y), a) f(x, y) \\
& =\sum_{y=a}^{\infty} \sum_{x=a}^{\infty}(1 \ominus p)^{\sigma(x)-a}(1 \ominus q)^{\sigma(y)-a} f(x, y) \\
& =\sum_{y=a}^{\infty} \sum_{x=a}^{\infty} \frac{f(x, y)}{(p+1)^{x+1-a}(q+1)^{y+1-a}} .
\end{aligned}
$$

In preceding steps, we use the definition of delta exponential function and the fact that $1 \ominus p=1 /(1+p)$ and $1 \ominus q=1 /(1+q)$, since $p$ and $q$ are regressive functions. In the following step, we use $x-a=j$ and $y-a=k$ to reindex the sums as follows:

$$
\mathscr{L}_{2}[f(x, y)]=\sum_{k=0}^{\infty} \sum_{j=0}^{\infty} \frac{f(a+j, a+k)}{(p+1)^{j+1}(q+1)^{k+1}} .
$$

Theorem 1. Assume functions $f(x, y): \mathbb{N}_{a} \times \mathbb{N}_{a} \longrightarrow \mathbb{R}$, $g(x): \mathbb{N}_{a} \longrightarrow \mathbb{R}$, and $h(y): \mathbb{N}_{a} \longrightarrow \mathbb{R}$ such that the delta double Laplace transforms exist, then the following holds:

$$
\begin{aligned}
& \text { (i) } \mathscr{L}_{2}\{g(x)\}(p, q)=(1 / q) \mathscr{L}_{x}\{g(x)\}(p) \\
& \text { (ii) } \mathscr{L}_{2}\{h(y)\}(p, q)=(1 / p) \mathscr{L}_{y}\{h(y)\}(q) \\
& \text { (iii) For } f(x, y)=g(x) h(y), \mathscr{L}_{2}\{f(x, y)\}(p, q)= \\
& \mathscr{L}_{x}\{g(x)\}(p) \mathscr{L}_{y}\{h(y)\}(q)
\end{aligned}
$$

Proof. Under the assumption stated above and by Lemma 4,

(i) For $p \neq-1$ and $q \neq 0,-1$, we have

$$
\begin{aligned}
\mathscr{L}_{2}\{g(x)\}(p, q) & =\sum_{k=0}^{\infty} \sum_{j=0}^{\infty} \frac{g(a+j)}{(p+1)^{j+1}(q+1)^{k+1}} \\
& =\sum_{k=0}^{\infty} \frac{1}{(q+1)^{k+1}} \sum_{j=0}^{\infty} \frac{g(a+j)}{(p+1)^{j+1}} \\
& =\frac{1}{q} \mathscr{L}_{x}\{g(x)\}(p) .
\end{aligned}
$$

(ii) The proof is similar to part (i).

(iii) For $p \neq-1$ and $q \neq-1$, we have

$$
\begin{aligned}
\mathscr{L}_{2}\{f(x, y)\}(p, q) & =\sum_{k=0}^{\infty} \sum_{j=0}^{\infty} \frac{g(a+j) h(a+k)}{(p+1)^{j+1}(q+1)^{k+1}} \\
& =\sum_{j=0}^{\infty} \frac{g(a+j)}{(p+1)^{j+1}} \sum_{k=0}^{\infty} \frac{h(a+k)}{(q+1)^{k+1}} \\
& =\mathscr{L}_{x}\{g(x)\}(p) \mathscr{L}_{y}\{h(y)\}(q) .
\end{aligned}
$$

Example 3

(i) If $f(x, y)=1$ for $x, y \in \mathbb{N}_{a}$, then $\mathscr{L}_{2}\{1\}=1 / p q$,

(ii) If $f(x, y)=(x-a)^{\underline{m}}(y-a)^{\underline{n}}$ for $x, y \in \mathbb{N}_{a}$, then $\mathscr{L}_{2}\left\{(x-a)^{\underline{m}}(y-a)^{\underline{n}}\right\}=(m ! n !) /\left(p^{m+1} q^{n+1}\right)$.

(iii) By Lemma 4

$$
\begin{aligned}
\mathscr{L}_{2}\{1\} & =\sum_{k=0}^{\infty} \sum_{j=0}^{\infty} \frac{1}{(p+1)^{j+1}(q+1)^{k+1}} \\
& =\sum_{k=0}^{\infty} \frac{1}{(q+1)^{k+1}} \sum_{j=0}^{\infty} \frac{1}{(p+1)^{j+1}} \\
& =\frac{1}{p q}, \quad \text { for } p, q \neq 0,-1 .
\end{aligned}
$$

(iv) By using Theorem 1 part (iii), we obtain

$$
\mathscr{L}_{2}\left\{(x-a)^{\underline{m}}(y-a)^{\underline{n}}\right\}=\mathscr{L}_{x}\left\{(x-a)^{\underline{m}}\right\} \mathscr{L}_{y}\left\{(y-a)^{\underline{n}}\right\} .
$$

By using Lemma 3,

$$
\begin{aligned}
& =\frac{m !}{p^{m+1}} \mathscr{L}_{y}\left\{(y-a)^{\frac{n}{}}\right\} \\
& =\frac{m !}{p^{m+1}} \frac{n !}{q^{n+1}} .
\end{aligned}
$$

If we choose either $m=0$ or $n=0$, then as a special case of the above

$$
\begin{aligned}
& \mathscr{L}_{2}\left\{(y-a)^{\underline{n}}\right\}=\frac{n !}{p q^{n+1}}, \quad \text { for } p, q \neq 0,-1, \\
& \mathscr{L}_{2}\left\{(x-a)^{\underline{m}}\right\}=\frac{m !}{p^{m+1} q}, \quad \text { for } p, q \neq 0,-1 .
\end{aligned}
$$

Coon and Bernstein $[20,21]$ defined the double Laplace transforms and discussed convergence and existence for the continuous case. We discuss discrete analogue of the double Laplace transforms. 
Definition 10. A function $f(x, y): \mathbb{N}_{a} \times \mathbb{N}_{a} \longrightarrow \mathbb{R}$ is of exponential order $r_{1}, r_{2}>0$ with respect to $x$ and $y$, respectively, if there exist a constant $A>0$ and $m, n \in \mathbb{N}_{0}$ such that, for each $x \in \mathbb{N}_{a+m}$ and $y \in \mathbb{N}_{a+n}$, the inequality $|f(x, y)| \leq A r_{1}^{x} r_{2}^{y}$ holds, where $A=\max \left\{A_{1}, A_{2}\right\}$ for $|f(x, a)| \leq A_{1} r_{1}^{x}$ and $|f(a, y)| \leq A_{2} r_{2}^{y}$.

Theorem 2. If a function $f(x, y): \mathbb{N}_{a} \times \mathbb{N}_{a} \longrightarrow \mathbb{R}$ is of exponential order $r_{1}, r_{2}>0$, then the delta double Laplace transform $\mathscr{L}_{2}\{f\}(p, q)$ converges absolutely for $p$ and $q$ provided $|p+1|>r_{1},|q+1|>r_{2}$.

Proof. Assume $f(x, y): \mathbb{N}_{a} \times \mathbb{N}_{a} \longrightarrow \mathbb{R}$ is of exponential order $r_{1}, r_{2}>0$. Then, there exists a constant $A>0$ and $m, n \in \mathbb{N}_{0}$ such that, for each $x \in \mathbb{N}_{a+m}$ and $y \in \mathbb{N}_{a+n}$, $|f(x, y)| \leq A r_{1}^{x} r_{2}^{y}$. Thus, for $|p+1|>r_{1}$ and $|q+1|>r_{2}$, we consider the following:

$$
\begin{aligned}
& \sum_{k=n}^{\infty} \sum_{j=m}^{\infty}\left|\frac{f(a+j, a+k)}{(p+1)^{j+1}(q+1)^{k+1}}\right| \\
& \leq \sum_{k=n}^{\infty} \sum_{j=m}^{\infty} \frac{A r_{1}^{j+a} r_{2}^{k+a}}{|p+1|^{j+1}|q+1|^{k+1}} \\
& \quad=\frac{A r_{1}^{a} r_{2}^{a}}{|p+1||q+1|} \sum_{k=n}^{\infty} \sum_{j=m}^{\infty}\left(\frac{r_{1}}{|p+1|}\right)^{j}\left(\frac{r_{2}}{|q+1|}\right)^{k} \\
& =\frac{A r_{1}^{a} r_{2}^{a}}{|p+1||q+1|} \frac{\left(r_{1} /|p+1|\right)^{m}}{\left(1-\left(r_{1} /|p+1|\right)\right)} \frac{\left(r_{2} /|q+1|\right)^{n}}{\left(1-\left(r_{2} /|q+1|\right)\right)} \\
& \quad=\frac{A r_{1}^{a+m} r_{2}^{a+n}}{|p+1|^{m}|q+1|^{n}\left[\left(|p+1|-r_{1}\right)\left(|q+1|-r_{2}\right)\right]} \\
& <\infty .
\end{aligned}
$$

Since $|p+1|>r_{1}$ and $|q+1|>r_{2}, \quad$ therefore $|p+1|-r_{1}>0$ and $|q+1|-r_{2}>0$. Hence, the delta double Laplace transform of $f$ converges absolutely.

Theorem 2 ensures the existence of the delta double Laplace transform. In general, the converse does not hold. We should consider functions $f$ of some exponential order $r>0$, to ensure the delta double Laplace transform of $f$ which does converge somewhere in the complex plane outside the both closed balls of radius $r_{1}, r_{2}$, centered at -1 , that is, we can choose $r=\max \left\{r_{1}, r_{2}\right\}$ for $|p+1|>r_{1}$ and $|q+1|>r_{2}$.

Theorem 3. Suppose $f, g: \mathbb{N}_{a} \times \mathbb{N}_{a} \longrightarrow \mathbb{R}$. If the delta double Laplace transform of $f, g$ converges for $|p+1|>r_{1}$ and $|q+1|>r_{2}$, where $r_{1}, r_{2}>0$, and let $c_{1}, c_{2} \in \mathbb{C}$, then the delta double Laplace transform of $c_{1} f+c_{2} g$ converges for $|p+1|>r_{1},|q+1|>r_{2}$, and $\mathscr{L}_{2}\left\{c_{1} f+c_{2} g\right\}(p, q)=c_{1} \mathscr{L}_{2}\{f\}$ $(p, q)+c_{2} \mathscr{L}_{2}\{g\}(p, q)$, converges for $|p+1|>r_{1}$ and $|q+1|>r_{2}$.

Proof. Since $f, g: \mathbb{N}_{a} \times \mathbb{N}_{a} \longrightarrow \mathbb{R}$ and the delta double Laplace transform of $f, g$ converges for $|p+1|>r_{1}$ and $|q+1|>r_{2}$, where $r_{1}, r_{2}>0$. We have that, for $|p+1|>r_{1}$ and $|q+1|>r_{2}$,

$$
\begin{aligned}
c_{1} \mathscr{L}_{2}\{f\}(p, q)+c_{2} \mathscr{L}_{2}\{g\}(p, q) \\
=c_{1} \sum_{k=0}^{\infty} \sum_{j=0}^{\infty} \frac{f(a+j, a+k)}{(p+1)^{j+1}(q+1)^{k+1}} \\
+c_{2} \sum_{k=0}^{\infty} \sum_{j=0}^{\infty} \frac{g(a+j, a+k)}{(p+1)^{j+1}(q+1)^{k+1}} \\
=\sum_{k=0}^{\infty} \sum_{j=0}^{\infty} \frac{\left(c_{1} f+c_{2} g\right)(a+j, a+k)}{(p+1)^{j+1}(q+1)^{k+1}} \\
=\mathscr{L}_{2}\left\{c_{1} f+c_{2} g\right\}(p, q) .
\end{aligned}
$$

Theorem 3 exposed the linearity property of the delta double Laplace transform, and Theorem 4 revealed the uniqueness.

Theorem 4. Suppose $f, g: \mathbb{N}_{a} \times \mathbb{N}_{a} \longrightarrow \mathbb{R}$ and $r_{1}>0, r_{2}>0$. If $\mathscr{L}_{2}\{f\}(p, q)=\mathscr{L}_{2}\{g\}(p, q)$, provided $|p+1|>r_{1}$, $|q+1|>r_{2}$, and $p, q \neq 0,-1$, then $f(x, y)=g(x, y)$ for all $x, y \in \mathbb{N}_{a}$.

Proof. By hypothesis, we have

$$
\mathscr{L}_{2}\{f\}(p, q)=\mathscr{L}_{2}\{g\}(p, q),
$$

for $|p+1|>r_{1}$ and $|q+1|>r_{2}$. This implies that

$$
\sum_{k=0}^{\infty} \sum_{j=0}^{\infty} \frac{f(a+j, a+k)}{(p+1)^{j+1}(q+1)^{k+1}}=\sum_{k=0}^{\infty} \sum_{j=0}^{\infty} \frac{g(a+j, a+k)}{(p+1)^{j+1}(q+1)^{k+1}},
$$

for $|p+1|>r_{1}$ and $|q+1|>r_{2}$. Since, by Theorem 2 , the double infinite series is absolute convergent, therefore comparison of both sides implies that

$$
f(a+j, a+k)=g(a+j, a+k), \quad \text { for all } j, k \in \mathbb{N}_{0} \text {. }
$$

For each fix $j$ and for all $y \in \mathbb{N}_{a}$, this implies that

$$
f(a+j, y)=g(a+j, y) .
$$

For each fix $k$, we obtain

$$
f(x, y)=g(x, y), \quad \text { for all } x, y \in \mathbb{N}_{a} .
$$

\section{Basic Properties of the Delta Double Laplace Transform}

In this section, following Bohner et al. [30], we prove some properties of the delta double Laplace transform. We also define double convolution product of discrete functions followed by Goodrich and Peterson [10] convolution product (single) of discrete functions. We present, the delta double Laplace transform of double convolution product for later use to solve difference equations. 
Theorem 5. Assume $f: \mathbb{N}_{a} \times \mathbb{N}_{a} \longrightarrow \mathbb{R}$ and $\mathscr{L}_{2}[f(x, y)]$ exists. If $\mathscr{L}_{2}[f(x, y)]=\widetilde{F}(p, q)$, then

$$
\begin{aligned}
& \mathscr{L}_{2} {[f(x-\alpha, y-\beta) H(x-\alpha, y-\beta)] } \\
&=e_{\ominus p}(\alpha, 0) e_{\ominus q}(\beta, 0)\left[\widetilde{\widetilde{F}}(p, q)-\sum_{s=0}^{c-a-1} \sum_{\tau=0}^{c-a-1} \frac{f(a+\tau, a+s)}{(p+1)^{\tau+1}(q+1)^{s+1}}\right],
\end{aligned}
$$

where $H(x, y)$ is the Heaviside unit step function defined by

$$
H(x-\alpha, y-\beta)= \begin{cases}0, & \text { if } x-\alpha, y-\beta \in \mathbb{N}_{a}^{c-1}, \\ 1, & \text { if } x-\alpha, y-\beta \in \mathbb{N}_{c} .\end{cases}
$$

Proof. We have, by Lemma 4,

$$
\begin{aligned}
\mathscr{L}_{2} & {[f(x-\alpha, y-\beta) H(x-\alpha, y-\beta)] } \\
= & \sum_{k=0}^{\infty} \sum_{j=0}^{\infty} \frac{f(a-\alpha+j, a-\beta+k) H(a-\alpha+j, a-\beta+k)}{(p+1)^{j+1}(q+1)^{k+1}} \\
& =\sum_{k=\beta+c-a}^{\infty} \sum_{j=\alpha+c-a}^{\infty} \frac{f(a-\alpha+j, a-\beta+k)}{(p+1)^{j+1}(q+1)^{k+1}} .
\end{aligned}
$$

Reindex by $j-\alpha=\tau$ and $k-\beta=s$,

$$
\begin{aligned}
& =\sum_{s=c-a}^{\infty} \sum_{\tau=c-a}^{\infty} \frac{f(a+\tau, a+s)}{(p+1)^{\alpha+\tau+1}(q+1)^{\beta+s+1}} \\
& =\frac{1}{(p+1)^{\alpha}(q+1)^{\beta}}\left[\sum_{s=0}^{\infty} \sum_{\tau=0}^{\infty} \frac{f(a+\tau, a+s)}{(p+1)^{\tau+1}(q+1)^{s+1}}-\sum_{s=0}^{c-a-1} \sum_{\tau=0}^{c-a-1} \frac{f(a+\tau, a+s)}{(p+1)^{\tau+1}(q+1)^{s+1}}\right] \\
& \mathscr{L}_{2}[f(x-\alpha, y-\beta) H(x-\alpha, y-\beta)]=e_{\ominus p}(\alpha, 0) e_{\ominus q}(\beta, 0)\left[\tilde{\widetilde{F}}(p, q)-\sum_{s=0}^{c-a-1} \sum_{\tau=0}^{c-a-1} \frac{f(a+\tau, a+s)}{(p+1)^{\tau+1}(q+1)^{s+1}}\right] .
\end{aligned}
$$

In the last step, we use Lemma 4 with the fact $e_{\ominus p}(\alpha, 0)=$ $1 /(p+1)^{\alpha}$ and $e_{\ominus q}(\beta, 0)=1 /(q+1)^{\beta}$.

Theorem 5 gives different results from its continuous counterpart stated in [23]. We state the useful shifting Theorem 6 for discrete setting.

Theorem 6. Assume $f: \mathbb{N}_{a} \times \mathbb{N}_{a} \longrightarrow \mathbb{R}$ and $\mathscr{L}_{2}[f(x, y)]$ exist. If $\mathscr{L}_{2}[f(x, y)]=\widetilde{F}(p, q)$, then

(i) $\mathscr{L}_{2}[f(x \underset{\widetilde{\widetilde{F}}}{\tilde{\mathrm{N}}}(\mathrm{c}-a), y-(c-a)) H(x, y)]=1 /[(p+1)$ $(q+1)]^{c-a} \widetilde{F}(p, q)$.

(ii) $\mathscr{L}_{2}[f(x+(c-a), y+(c-a))]=\left[\begin{array}{ll}(p+1) & (q+1)\end{array}\right]^{c-a}$ $\left[\widetilde{F}(p, q)-\sum_{s=0}^{c-a-1} \sum_{\tau=0}^{c-a-1} f(a+\tau, a+s) /\left((p+1)^{\tau+1}\right.\right.$ $\left.\left.(q+1)^{s+1}\right)\right]$

where $H(x, y)$ is the Heaviside unit step function defined by

$$
H(x, y)= \begin{cases}0, & \text { if } x, y \in \mathbb{N}_{a}^{c-1}, \\ 1, & \text { if } x, y \in \mathbb{N}_{c} .\end{cases}
$$

Proof

(i) We have by Lemma $4, \mathscr{L}_{2}[f(x-(c-a)$, $y-(c-a)) H(x, y)]$

$$
\begin{array}{r}
=\sum_{k=0}^{\infty} \sum_{j=0}^{\infty} \frac{f(j+2 a-c, k+2 a-c) H(a+j, a+k)}{(p+1)^{j+1}(q+1)^{k+1}} \\
=\sum_{k=c-a}^{\infty} \sum_{j=c-a}^{\infty} \frac{f(j+2 a-c, k+2 a-c)}{(p+1)^{j+1}(q+1)^{k+1}} .
\end{array}
$$

Reindex by $\tau=j+a-c$ and $s=k+a-c$,

$$
\begin{aligned}
& =\sum_{s=0}^{\infty} \sum_{\tau=0}^{\infty} \frac{f(a+\tau, a+s)}{(p+1)^{\tau+c-a+1}(q+1)^{s+c-a+1}} \\
& =\frac{1}{[(p+1)(q+1)]^{c-a}} \sum_{s=0}^{\infty} \sum_{\tau=0}^{\infty} \frac{f(a+\tau, a+s)}{(p+1)^{\tau+1}(q+1)^{s+1}} \\
& =\frac{1}{[(p+1)(q+1)]^{c-a}} \widetilde{\widetilde{F}}(p, q) .
\end{aligned}
$$

(ii) By use of Lemma 4 and reindex by $\tau=j+c-a$ and $s=k+c-a$,

$$
\begin{aligned}
\mathscr{L}_{2} & {[f(x+(c-a), y+(c-a))] } \\
& =\sum_{k=0}^{\infty} \sum_{j=0}^{\infty} \frac{f(j+c, k+c)}{(p+1)^{j+1}(q+1)^{k+1}} \\
& =\sum_{s=c-a}^{\infty} \sum_{\tau=c-a}^{\infty} \frac{f(a+\tau, a+s)}{(p+1)^{\tau+a-c+1}(q+1)^{s+a-c+1}} \\
& =[(p+1)(q+1)]^{c-a} \sum_{s=c-a}^{\infty} \sum_{\tau=c-a}^{\infty} \frac{f(a+\tau, a+s)}{(p+1)^{\tau+1}(q+1)^{s+1}} \\
& =[(p+1)(q+1)]^{c-a}\left[\widetilde{\widetilde{F}}(p, q)-\sum_{s=0}^{c-a-1} \sum_{\tau=0}^{c-a-1} \frac{f(a+\tau, a+s)}{(p+1)^{\tau+1}(q+1)^{s+1}}\right] .
\end{aligned}
$$


Theorem 7. Assume $f(x, y)$ is periodic with $T_{1}, T_{2} \in \mathbb{N}_{1}$ and $\mathscr{L}_{2}[f(x, y)]$ exist; then,

$$
\mathscr{L}_{2}[f(x, y)]=\frac{1}{\left[1-e_{\ominus p}\left(T_{1}, 0\right) e_{\ominus q}\left(T_{2}, 0\right)\right]} \sum_{j=0}^{T_{1}-1} \sum_{k=0}^{T_{2}-1} \frac{f(a+j, a+k)}{(p+1)^{j+1}(q+1)^{k+1}}
$$

Proof. Under the assumption, we have, by Lemma 4,

$$
\begin{aligned}
\mathscr{L}_{2}[f(x, y)] & =\sum_{k=0}^{\infty} \sum_{j=0}^{\infty} \frac{f(a+j, a+k)}{(p+1)^{j+1}(q+1)^{k+1}} \\
& =\sum_{k=0}^{T_{2}-1} \sum_{j=0}^{T_{1}-1} \frac{f(a+j, a+k)}{(p+1)^{j+1}(q+1)^{k+1}}+\sum_{k=T_{2}}^{\infty} \sum_{j=T_{1}}^{\infty} \frac{f(a+j, a+k)}{(p+1)^{j+1}(q+1)^{k+1}} \\
& =\sum_{k=0}^{T_{2}-1} \sum_{j=0}^{T_{1}-1} \frac{f(a+j, a+k)}{(p+1)^{j+1}(q+1)^{k+1}}+\sum_{v=0}^{\infty} \sum_{u=0}^{\infty} \frac{f\left(a+u+T_{1}, a+v+T_{2}\right)}{(p+1)^{T_{1}+u+1}(q+1)^{T_{2}+v+1}} .
\end{aligned}
$$

In the last step, we used $j=T_{1}+u$ and $k=T_{2}+v$ to reindex second double summation. In second double summation, periodicity of $f$ implies that

$$
\begin{aligned}
\mathscr{L}_{2}[f(x, y)] & =\sum_{k=0}^{T_{2}-1} \sum_{j=0}^{T_{1}-1} \frac{f(a+j, a+k)}{(p+1)^{j+1}(q+1)^{k+1}}+\sum_{v=0}^{\infty} \sum_{u=0}^{\infty} \frac{f(a+u, a+v)}{(p+1)^{T_{1}+u+1}(q+1)^{T_{2}+v+1}} \\
& =\sum_{k=0}^{T_{2}-1} \sum_{j=0}^{T_{1}-1} \frac{f(a+j, a+k)}{(p+1)^{j+1}(q+1)^{k+1}}+\left[\frac{1}{(p+1)}\right]^{T_{1}}\left[\frac{1}{(q+1)}\right]^{T_{2}} \sum_{v=0}^{\infty} \sum_{u=0}^{\infty} \frac{f(a+u, a+v)}{(p+1)^{u+1}(q+1)^{v+1}} \\
& =\sum_{k=0}^{T_{2}-1} \sum_{j=0}^{T_{1}-1} \frac{f(a+j, a+k)}{(p+1)^{j+1}(q+1)^{k+1}}+e_{\ominus p}\left(T_{1}, 0\right) e_{\ominus q}\left(T_{2}, 0\right) \sum_{v=0}^{\infty} \sum_{u=0}^{\infty} \frac{f(a+u, a+v)}{(p+1)^{u+1}(q+1)^{v+1}} \\
& =\sum_{k=0}^{T_{2}-1} \sum_{j=0}^{T_{1}-1} \frac{f(a+j, a+k)}{(p+1)^{j+1}(q+1)^{k+1}}+e_{\ominus p}\left(T_{1}, 0\right) e_{\ominus q}\left(T_{2}, 0\right) \mathscr{L}_{2}[f(x, y)] \\
& =\frac{1}{\left[1-e_{\ominus p}\left(T_{1}, 0\right) e_{\ominus q}\left(T_{2}, 0\right)\right]} \sum_{k=0}^{T_{2}-1} \sum_{j=0}^{T_{1}-1} \frac{f(a+j, a+k)}{(p+1)^{j+1}(q+1)^{k+1}} .
\end{aligned}
$$

Definition 11. Assume $f, g: \mathbb{N}_{a} \times \mathbb{N}_{a} \longrightarrow \mathbb{R}$. The double convolution product is defined by

$$
\begin{aligned}
(f * * g)(x, y)= & \sum_{r=a}^{x-1} \sum_{s=a}^{y-1} f(r, s) g(x-\sigma(r)+a, \\
& y-\sigma(s)+a) \text { for } x, y \in \mathbb{N}_{a} .
\end{aligned}
$$

Note, by empty sum convention, $(f * * g)(a, a)=0$.
Lemma 5. Assume $f, g: \mathbb{N}_{a} \times \mathbb{N}_{a} \longrightarrow \mathbb{R}$. The double convolution product is commutative:

$$
(f * * g)(x, y)=(g * * f)(x, y) \text { for } x, y \in \mathbb{N}_{a} .
$$

Proof. By Definition 11 and the change of variables $x-r-$ $1+a=u$ and $y-s-1+a=v$, we have 


$$
\begin{aligned}
(g * * f)(x, y) & =\sum_{r=a}^{x-1} \sum_{s=a}^{y-1} g(r, s) f(x-\sigma(r)+a, y-\sigma(s)+a) \\
& \text { for } x, y \in \mathbb{N}_{a}, \\
& =\sum_{u=a}^{x-1} \sum_{v=a}^{y-1} g(x-\sigma(u)+a, y-\sigma(v)+a) f(u, v), \\
& =(f * * g)(x, y), \quad \text { for } x, y \in \mathbb{N}_{a} .
\end{aligned}
$$

Theorem 8 (convolution theorem). Assume $f, g: \mathbb{N}_{a} \times$ $\mathbb{N}_{a} \longrightarrow \mathbb{R}$. If both $\mathscr{L}_{2}[f(x, y)]$ and $\mathscr{L}_{2}[g(x, y)]$ exist, then the delta double Laplace transform of double convolution product is

$$
\mathscr{L}_{2}\{(f * * g)(x, y)\}=\mathscr{L}_{2}\{f(x, y)\} \mathscr{L}_{2}\{g(x, y)\} .
$$

Proof. Under given assumption, we have, by Lemma 4 and the fact $(f * * g)(a, a)=0$,

$$
\begin{aligned}
& \mathscr{L}_{2}\{(f * * g)(x, y)\}=\sum_{k=0}^{\infty} \sum_{j=0}^{\infty} \frac{(f * * g)(a+j, a+k)}{(p+1)^{j+1}(q+1)^{k+1}} \\
&=\sum_{k=1}^{\infty} \sum_{j=1}^{\infty} \frac{(f * * g)(a+j, a+k)}{(p+1)^{j+1}(q+1)^{k+1}} \\
&=\sum_{k=1}^{\infty} \sum_{j=1}^{\infty} \frac{1}{(p+1)^{j+1}(q+1)^{k+1}} \\
& \sum_{r=a}^{a+k-1} \sum_{s=a}^{a+j-1} f(r, s) g(a+j-\sigma(r)+a, a+k-\sigma(s)+a) .
\end{aligned}
$$

In the last step, we used Definition 11; next, making the change of variables $r \longrightarrow a+r$ and $s \longrightarrow a+s$ gives us that

$$
\begin{aligned}
& =\sum_{k=1}^{\infty} \sum_{j=1}^{\infty} \sum_{r=0}^{k-1} \sum_{s=0}^{j-1} \frac{f(a+r, a+s) g(a+j-\sigma(r), a+k-\sigma(s))}{(p+1)^{j+1}(q+1)^{k+1}} \\
& =\sum_{k=1}^{\infty} \sum_{r=0}^{k-1} \sum_{j=1}^{\infty} \sum_{s=0}^{j-1} \frac{f(a+r, a+s) g(a+j-\sigma(r), a+k-\sigma(s))}{(p+1)^{j+1}(q+1)^{k+1}} \\
& =\sum_{r=0}^{\infty} \sum_{k=1}^{\infty} \sum_{s=0}^{\infty} \sum_{j=1}^{\infty} \frac{f(a+r, a+s) g(a+j-\sigma(r), a+k-\sigma(s))}{(p+1)^{j+1}(q+1)^{k+1}} \\
& =\sum_{r=0}^{\infty} \sum_{\tau_{2}=0}^{\infty} \sum_{s=0}^{\infty} \sum_{\tau_{1}=0}^{\infty} \frac{f(a+r, a+s) g\left(a+\tau_{1}, a+\tau_{2}\right)}{(p+1)^{\tau_{1}+r+2}(q+1)^{\tau_{2}+s+2}} \\
& =\sum_{s=0}^{\infty} \sum_{r=0}^{\infty} \frac{f(a+r, a+s)}{(p+1)^{r+1}(q+1)^{s+1}} \sum_{\tau_{2}=0}^{\infty} \sum_{\tau_{1}=0}^{\infty} \frac{g\left(a+\tau_{1}, a+\tau_{2}\right)}{(p+1)^{\tau_{1}+1}(q+1)^{\tau_{2}+1}} \\
& =\mathscr{L}_{2}\{f(x, y)\} \mathscr{L}_{2}\{g(x, y)\} .
\end{aligned}
$$

In the previous steps, we interchanged the order of first pairs and second pairs of summation and change variables $j-r-1=\tau_{1}$ and $k-s-1=\tau_{2}$.

Corollary 1. Assume $f, g: \mathbb{N}_{a} \times \mathbb{N}_{a} \longrightarrow \mathbb{R}$. If $f(x, y)=u_{1}(x) v_{1}(y)$ and $g(x, y)=u_{2}(x) v_{2}(y)$ and the delta Laplace transform exists, then

$$
\mathscr{L}_{2}\{(f * * g)(x, y)\}=\mathscr{L}_{x}\left\{\left(u_{1} * u_{2}\right)(x)\right\} \mathscr{L}_{y}\left\{\left(v_{1} * v_{2}\right)(y)\right\},
$$

where the product on right-and left-hand sides is given by Definitions 5 and 11, respectively.

Proof. By double convolution theorem, we have

$$
\mathscr{L}_{2}\{(f * * g)(x, y)\}=\mathscr{L}_{2}\{f(x, y)\} \mathscr{L}_{2}\{g(x, y)\} .
$$

Since $\quad \mathscr{L}_{2}[f(x, y)]=\mathscr{L}_{2}\left[u_{1}(x) v_{1}(y)\right]=\mathscr{L}_{x}\left[u_{1}(x)\right]$ $\mathscr{L}_{y}\left[v_{1}(y)\right]$ and $\mathscr{L}_{2}[g(x, y)]=\mathscr{L}_{2}\left[u_{2}(x) v_{2}(y)\right]=\mathscr{L}_{x}\left[u_{2}(x)\right]$ $\mathscr{L}_{y}\left[v_{2}(y)\right]$,

$$
\begin{aligned}
& \text { consider } \mathscr{L}_{2}\{(f * * g)(x, y)\} \\
& =\mathscr{L}_{x}\left[u_{1}(x)\right] \mathscr{L}_{y}\left[v_{1}(y)\right] \mathscr{L}_{x}\left[u_{2}(x)\right] \mathscr{L}_{y}\left[v_{2}(y)\right] \\
& =\mathscr{L}_{x}\left[u_{1}(x)\right] \mathscr{L}_{x}\left[u_{2}(x)\right] \mathscr{L}_{y}\left[v_{1}(y)\right] \mathscr{L}_{y}\left[v_{2}(y)\right] \\
& =\mathscr{L}_{x}\left\{\left(u_{1} * u_{2}\right)(x)\right\} \mathscr{L}_{y}\left\{\left(v_{1} * v_{2}\right)(y)\right\} .
\end{aligned}
$$

The last step is followed from single convolution Lemma 1.

\section{The Delta Double Laplace Transforms of Partial Differences}

In this section, we examine the action of the delta double Laplace transforms on first order partial differences. The results developed for first order partial differences are further used to establish properties of the delta double Laplace transforms of generalized order partial difference, similar to that appeared in [40] for fractional order partial derivatives. We usually consider functions $u(x, y): \mathbb{N}_{a} \times \mathbb{N}_{a} \longrightarrow \mathbb{R}$, of exponential order $r_{1}, r_{2}>0$ with respect to $x$ and $y$, respectively, ensuring that delta Laplace and the delta double Laplace transforms of $u(x, y)$ and its partial differences does exist.

Lemma 6. Assume $u(x, y): \mathbb{N}_{a} \times \mathbb{N}_{a} \longrightarrow \mathbb{R}$, such that the delta Laplace transforms exist for constants $p \neq-1$ and $q \neq-1$. Then,

$$
\begin{aligned}
& \mathscr{L}_{x} \Delta_{x}[u(x, y)]=p \mathscr{L}_{x}\{u(x, y)\}-u(a, y), \\
& \mathscr{L}_{y} \Delta_{y}[u(x, y)]=q \mathscr{L}_{y}\{u(x, y)\}-u(x, a), \\
& \mathscr{L}_{x} \Delta_{y}[u(x, y)]=\Delta_{y} \mathscr{L}_{x} u(x, y), \\
& \mathscr{L}_{y} \Delta_{x}[u(x, y)]=\Delta_{x} \mathscr{L}_{y} u(x, y) .
\end{aligned}
$$

Proof. By definition of the delta Laplace transforms on $x$, 


$$
\mathscr{L}_{x} \Delta_{x}[u(x, y)]=\int_{a}^{\infty} e_{\ominus p}(\sigma(x), a) \Delta_{x} u(x, y) \Delta x .
$$

Apply summation by parts (Lemma 2) on $x$, and using the fact $\Delta_{x}\left[e_{\ominus p}(\sigma(x), a)\right]=\ominus p e_{\ominus p}(x, a)$, we have that

$$
=\left.e_{\ominus p}(x, a) u(x, y)\right|_{x=a} ^{\infty}-\int_{a}^{\infty} u(x, y)\left[\ominus p e_{\ominus p}(x, a)\right] \Delta x .
$$

Use the fact $e_{\ominus p}(x, a)=1 /(p+1)^{x-a}$ and $\ominus p=-p /(p+1)$

$$
\begin{aligned}
& =\left.\frac{1}{(p+1)^{x-a}} u(x, y)\right|_{x=a} ^{\infty}-\int_{a}^{\infty} u(x, y)\left(\frac{-p}{(p+1)}\right) e_{\ominus p}(x, a) \Delta x . \\
& \text { Since }(p+1) e_{\ominus p}(\sigma(x), a)=e_{\ominus p}(x, a), \\
& =[0-u(a, y)]+p \int_{a}^{\infty} u(x, y) e_{\ominus p}(\sigma(x), a) \Delta x \\
& =-u(a, y)+p \mathscr{L}_{x}\{u(x, y)\}, \\
& \mathscr{L}_{x} \Delta_{x}[u(x, y)]=p \mathscr{L}_{x}\{u(x, y)\}-u(a, y) .
\end{aligned}
$$

Let $\mathscr{L}_{x} u(x, y)=\widetilde{u}(p, y)$. Consider the left-hand side of equation (61) and use the definition of delta difference:

$$
\mathscr{L}_{x} \Delta_{y}[u(x, y)]=\mathscr{L}_{x}[u(x, y+1)-u(x, y)] .
$$

By using linearity property of the delta Laplace transforms, we obtain

$$
\begin{aligned}
& =\mathscr{L}_{x} u(x, y+1)-\mathscr{L}_{x} u(x, y) \\
& =\widetilde{u}(p, y+1)-\widetilde{u}(p, y) .
\end{aligned}
$$

Now, consider the right-hand side of equation (61) and use $\mathscr{L}_{x} u(x, y)=\widetilde{u}(p, y)$ :

$$
\Delta_{y} \mathscr{L}_{x}[u(x, y)]=\Delta_{y} \widetilde{u}(p, y) .
$$

By using the definition of delta difference, we obtain

$$
=\widetilde{u}(p, y+1)-\widetilde{u}(p, y) .
$$

Equality holds in equation (61) from equations (68) and (70). Proof of equations (60) and (62) is similar to proof of equations (59) and (61), respectively.

Theorem 9. Assume $u(x, y): \mathbb{N}_{a} \times \mathbb{N}_{a} \longrightarrow \mathbb{R}$, such that the delta double Laplace transforms exist for constants $p \neq-1$ and $q \neq-1$. Then,

$$
\begin{aligned}
\text { (i) } \mathscr{L}_{2} \Delta_{x}[u(x, y)] & =p \mathscr{L}_{2}\{u(x, y)\}-\mathscr{L}_{y}\{u(a, y)\} \\
\text { (ii) } \mathscr{L}_{2} \Delta_{y}[u(x, y)] & =q \mathscr{L}_{2}\{u(x, y)\}-\mathscr{L}_{x}\{u(x, a)\}
\end{aligned}
$$

Proof. Since, by definition, the delta double Laplace transforms is the successive application of the delta Laplace transforms on $x$ and $y$ in any order, therefore $\mathscr{L}_{2}=\mathscr{L}_{x} \mathscr{L}_{y}=\mathscr{L}_{y} \mathscr{L}_{x}$.

(i) Consider

$$
\mathscr{L}_{2} \Delta_{x}[u(x, y)]=\mathscr{L}_{y}\left[\mathscr{L}_{x} \Delta_{x} u(x, y)\right] .
$$

By using equation (59) of Lemma 6, we obtain

$$
=\mathscr{L}_{y}\left[p \mathscr{L}_{x}\{u(x, y)\}-u(a, y)\right] .
$$

Use linearity property of the delta Laplace transforms for $\mathscr{L}_{y}$,

$$
=p \mathscr{L}_{2}\{u(x, y)\}-\mathscr{L}_{y}[u(a, y)] .
$$

(ii) The proof is similar to part (i).

Note that, for constant $a, \Delta_{x}\{u(a, y)\}=u(a, y)$ $u(a, y)=0$. We adopt the following symbols in our result which are nonzero, in general, $\Delta_{x}\{u(a, y)\}=$ $\left.\Delta_{x}\{u(x, y)\}\right|_{x=a}$ and $\Delta_{y}\{u(x, a)\}=\left.\Delta_{y}\{u(x, y)\}\right|_{y=a}$, that is, first we take difference and then evaluate at $a$.

Lemma 7. Assume $u(x, y): \mathbb{N}_{a} \times \mathbb{N}_{a} \longrightarrow \mathbb{R}$, such that the delta Laplace transforms exist for constants $p \neq-1$ and $q \neq-1$. Then,

(i) $\mathscr{L}_{x} \Delta_{x}^{n}[u(x, y)]=p^{n} \mathscr{L}_{x}\{u(x, y)\}-\sum_{k=0}^{n-1} p^{n-1-k} \Delta_{x}^{k} u$ $(a, y)$

(ii) $\mathscr{L}_{y} \Delta_{y}^{m}[u(x, y)]=q^{m} \mathscr{L}_{y}\{u(x, y)\}-\sum_{j=0}^{m-1} q^{m-1-j}$ $\Delta_{y}^{j} u(x, a)$

(iii) $\mathscr{L}_{x} \Delta_{x y}^{n m}[u(x, y)]=p^{n} \mathscr{L}_{x} \Delta_{y}^{m}\{u(x, y)\}-\sum_{k=0}^{n-1} p^{n-1-k}$ $\Delta_{x}^{k} \Delta_{y}^{m} u(a, y)$

(iv) $\mathscr{L}_{y} \Delta_{x y}^{n m}[u(x, y)]=q^{m} \mathscr{L}_{y} \Delta_{x}^{n}\{u(x, y)\}-\sum_{j=0}^{m-1} q^{m-1-j}$ $\Delta_{x}^{n} \Delta_{y}^{j} \mathcal{u}(x, a)$

Proof

(i) We prove this part by induction on $n$, and result for $n=1$ has been proved in Lemma 6. Assume the result is true for $n \geq 1$ :

$$
\mathscr{L}_{x} \Delta_{x}^{n}[u(x, y)]=p^{n} \mathscr{L}_{x}\{u(x, y)\}-\sum_{k=0}^{n-1} p^{n-1-k} \Delta_{x}^{k} u(a, y) .
$$

We will try to establish result for $n+1$, beginning with the following:

$$
\mathscr{L}_{x} \Delta_{x}^{n+1}[u(x, y)]=\mathscr{L}_{x}\left[\Delta_{x} \Delta_{x}^{n} u(x, y)\right] .
$$

Let $w(x, y)=\Delta_{x}^{n}[u(x, y)]$, and we have that

$$
=\mathscr{L}_{x}\left[\Delta_{x} w(x, y)\right] \text {. }
$$

Again using equation (59) of Lemma 6,

$$
\begin{aligned}
& \left.=p \mathscr{L}_{x}\{w(x, y)\}-w(a, y)\right] \\
& =p \mathscr{L}_{x}\left\{\Delta_{x}^{n}[u(x, y)]\right\}-\Delta_{x}^{n}[u(a, y)] .
\end{aligned}
$$

By using assumption for $n$,

$$
\begin{aligned}
& =p\left[p^{n} \mathscr{L}_{x}\{u(x, y)\}-\sum_{k=0}^{n-1} p^{n-1-k} \Delta_{x}^{k} u(a, y)\right]-\Delta_{x}^{n}[u(a, y)] \\
& =p^{n+1} \mathscr{L}_{x}\{u(x, y)\}-\sum_{k=0}^{n-1} p^{n-k} \Delta_{x}^{k} u(a, y)-p^{n-n} \Delta_{x}^{n}[u(a, y)] \\
& \mathscr{L}_{x} \Delta_{x}^{n+1}[u(x, y)]=p^{n+1} \mathscr{L}_{x}\{u(x, y)\}-\sum_{k=0}^{n} p^{n-k} \Delta_{x}^{k} u(a, y) .
\end{aligned}
$$


The result holds for $n+1$, whenever it holds for $n$. Hence, by induction, result in part (i) holds.

(ii)

$$
\mathscr{L}_{x} \Delta_{x y}^{n m}[u(x, y)]=\mathscr{L}_{x} \Delta_{x}^{n}\left[\Delta_{y}^{m} u(x, y)\right] .
$$

Let $v(x, y)=\Delta_{y}^{m} u(x, y)$, and use part (i) of the same Lemma:

$$
\begin{aligned}
& =\mathscr{L}_{x} \Delta_{x}^{n}[v(x, y)] \\
& =p^{n} \mathscr{L}_{x}\{v(x, y)\}-\sum_{k=0}^{n-1} p^{n-1-k} \Delta_{x}^{k} v(a, y) \\
& =p^{n} \mathscr{L}_{x} \Delta_{y}^{m}\{u(x, y)\}-\sum_{k=0}^{n-1} p^{n-1-k} \Delta_{x}^{k} \Delta_{y}^{m} u(a, y) .
\end{aligned}
$$

Proof of (ii) and (iv) is similar as proof of part (i) and (iii), respectively.
Theorem 10. Assume $u(x, y): \mathbb{N}_{a} \times \mathbb{N}_{a} \longrightarrow \mathbb{R}$, such that the delta double Laplace transforms exist for constants $p \neq-1$ and $q \neq-1$. Then,

(i) $\mathscr{L}_{2} \Delta_{x}^{n}[u(x, y)]=p^{n} \mathscr{L}_{2}\{u(x, y)\}-\sum_{k=0}^{n-1} p^{n-1-k} \mathscr{L}_{y} \times$ $\left\{\Delta_{x}^{k} u(a, y)\right\}$

(ii) $\mathscr{L}_{2} \Delta_{y}^{m}[u(x, y)]=q^{m} \mathscr{L}_{2}\{u(x, y)\}-\sum_{j=0}^{m-1} q^{m-1-j} \mathscr{L}_{x} \times$ $\left\{\Delta_{y}^{j} u(x, a)\right\}$

(iii) $\mathscr{L}_{2} \Delta_{x y}^{n m}[u(x, y)]=p^{n} q^{m}\left[\mathscr{L}_{2}\{u(x, y)\}-\sum_{k=0}^{n-1} p^{-1-k}\right.$ $\mathscr{L}_{y}\left\{\Delta_{x}^{k} u(a, y)\right\}-\sum_{j=0}^{m-1} q^{-1-j} \mathscr{L}_{x}\left\{\Delta_{y}^{j} u(x, a)\right\}+$ $\left.\sum_{j=0}^{m-1} \sum_{k=0}^{n-1} p^{-1-k} q^{-1-j}\left\{\Delta_{x y}^{k j} u(a, a)\right\}\right]$

Proof. Since by definition, the delta double Laplace transforms is the successive application of the delta Laplace transforms on $x$ and $y$ in any order; therefore, $\mathscr{L}_{2}=\mathscr{L}_{x} \mathscr{L}_{y}=\mathscr{L}_{y} \mathscr{L}_{x}$.

(i) Using Lemma 7 part (i) and linearity of Laplace, we consider the following:

$$
\begin{aligned}
\mathscr{L}_{2} \Delta_{x}^{n}[u(x, y)] & =\mathscr{L}_{y}\left[\mathscr{L}_{x} \Delta_{x}^{n} u(x, y)\right] \\
& =\mathscr{L}_{y}\left[p^{n} \mathscr{L}_{x}\{u(x, y)\}-\sum_{k=0}^{n-1} p^{n-1-k} \Delta_{x}^{k} u(a, y)\right] \\
& =p^{n} \mathscr{L}_{y} \mathscr{L}_{x}\{u(x, y)\}-\mathscr{L}_{y} \sum_{k=0}^{n-1} p^{n-1-k} \Delta_{x}^{k} u(a, y) \\
& =p^{n} \mathscr{L}_{2}\{u(x, y)\}-\sum_{k=0}^{n-1} p^{n-1-k} \mathscr{L}_{y}\left\{\Delta_{x}^{k} u(a, y)\right\} .
\end{aligned}
$$

(ii) Proof is similar as in part (i).

(iii) Using Lemma 7 part (iii) and linearity of Laplace, we consider the following:

$$
\begin{aligned}
\mathscr{L}_{2} \Delta_{x y}^{n m}[u(x, y)] & =\mathscr{L}_{y}\left[\mathscr{L}_{x} \Delta_{x y}^{n m} u(x, y)\right] \\
& =\mathscr{L}_{y}\left[p^{n} \mathscr{L}_{x} \Delta_{y}^{m}\{u(x, y)\}-\sum_{k=0}^{n-1} p^{n-1-k} \Delta_{x}^{k} \Delta_{y}^{m} u(a, y)\right] \\
& =p^{n}\left[\mathscr{L}_{y} \mathscr{L}_{x} \Delta_{y}^{m}\{u(x, y)\}\right]-p^{n} \sum_{k=0}^{n-1} p^{-1-k}\left[\mathscr{L}_{y} \Delta_{x}^{k} \Delta_{y}^{m} u(a, y)\right] \\
& =p^{n}\left[\mathscr{L}_{2} \Delta_{y}^{m}\{u(x, y)\}\right]-p^{n} \sum_{k=0}^{n-1} p^{-1-k}\left[q^{m} \mathscr{L}_{y} \Delta_{x}^{k}\{u(a, y)\}-\sum_{j=0}^{m-1} q^{m-1-j} \Delta_{x}^{k} \Delta_{y}^{j} u(a, a)\right] .
\end{aligned}
$$

In the previous step, we used Lemma 7 part (iv). In the following step, using Theorem 10 part (ii), 


$$
\begin{aligned}
= & p^{n}\left[q^{m} \mathscr{L}_{2}\{u(x, y)\}-\sum_{j=0}^{m-1} q^{m-1-j} \mathscr{L}_{y}\left\{\Delta_{y}^{j} u(x, a)\right\}\right] \\
& -p^{n} \sum_{k=0}^{n-1} p^{-1-k}\left[q^{m} \mathscr{L}_{y} \Delta_{x}^{k}\{u(a, y)\}-\sum_{j=0}^{m-1} q^{m-1-j} \Delta_{x}^{k} \Delta_{y}^{j} u(a, a)\right] \\
= & p^{n} q^{m}\left[\mathscr{L}_{2}\{u(x, y)\}-\sum_{k=0}^{n-1} p^{-1-k} \mathscr{L}_{y}\left\{\Delta_{x}^{k} u(a, y)\right\}-\sum_{j=0}^{m-1} q^{-1-j} \mathscr{L}_{x}\left\{\Delta_{y}^{j} u(x, a)\right\}+\sum_{j=0}^{m-1} \sum_{k=0}^{n-1} p^{-1-k} q^{-1-j}\left\{\Delta_{x y}^{k j} u(a, a)\right\}\right] .
\end{aligned}
$$

Theorem 11. Assume $f(x, y): \mathbb{N}_{a} \times \mathbb{N}_{a} \longrightarrow \mathbb{R}$. If $\mathscr{L}_{2}[f(x, y)]=\widetilde{\widetilde{F}}(p, q)$, then for constants $p \neq 0,-1$ and $q \neq 0,-1$, and we have

$$
\mathscr{L}_{2}\left[\int_{a}^{x} \int_{a}^{y} f(t, \tau) \Delta \tau \Delta t\right]=\frac{\widetilde{F}(p, q)}{p q}
$$

Proof. For $x, y \in \mathbb{N}_{a}$, let

$$
u(x, y)=\int_{a}^{x} \int_{a}^{y} f(t, \tau) \Delta \tau \Delta t .
$$

Then, the difference $\Delta_{x y}$ is

$$
\begin{aligned}
\Delta_{x y} u(x, y) & =\Delta_{y}\left[\Delta_{x} u(x, y)\right] \\
& =\Delta_{y}\left[\int_{a}^{x+1} \int_{a}^{y} f(t, \tau) \Delta \tau \Delta t-\int_{a}^{x} \int_{a}^{y} f(t, \tau) \Delta \tau \Delta t\right] \\
& =\Delta_{y}\left[\sum_{t=a}^{x} \sum_{\tau=a}^{y-1} f(t, \tau)-\sum_{t=a}^{x-1} \sum_{\tau=a}^{y-1} f(t, \tau)\right] .
\end{aligned}
$$

By separating last term for $t=x$, from the first double sum, we obtain

$$
\begin{aligned}
& =\Delta_{y}\left[\sum_{\tau=a}^{y-1} f(x, \tau)+\sum_{t=a}^{x-1} \sum_{\tau=a}^{y-1} f(t, \tau)-\sum_{t=a}^{x-1} \sum_{\tau=a}^{y-1} f(t, \tau)\right] \\
& =\Delta_{y}\left[\sum_{\tau=a}^{y-1} f(x, \tau)\right] \\
& =\sum_{\tau=a}^{y} f(x, \tau)-\sum_{\tau=a}^{y-1} f(x, \tau) .
\end{aligned}
$$

By separating last term for $\tau=y$, from the first sum, we obtain

$$
\begin{aligned}
& =f(x, y)+\sum_{\tau=a}^{y-1} f(x, \tau)-\sum_{\tau=a}^{y-1} f(x, \tau) \\
f(x, y) & =\Delta_{x y} u(x, y) .
\end{aligned}
$$

Now, for constants $p \neq 0,-1$ and $q \neq 0,-1$, taking the delta double Laplace transforms on both sides,

$$
\mathscr{L}_{2} f(x, y)=\mathscr{L}_{2} \Delta_{x y} u(x, y) .
$$

By application of Theorem 10 (iii) for $m=1=n$, $\mathscr{L}_{2} \Delta_{x y}[u(x, y)]=p q \mathscr{L}_{2}[u(x, y)]-q \mathscr{L}_{y}\{u(a, y)\}-p \mathscr{L}_{x}$ $\{u(x, a)\}+u(a, a)$. On right-hand side, we obtain

$$
\begin{aligned}
= & p q \mathscr{L}_{2}[u(x, y)]-q \mathscr{L}_{y}\{u(a, y)\} \\
& -p \mathscr{L}_{x}\{u(x, a)\}+u(a, a) \\
\mathscr{L}_{2} f(x, y)= & p q \mathscr{L}_{2}[u(x, y)] .
\end{aligned}
$$

In the last step, $u(x, a), u(a, y), u(a, a)$ are zero by empty sum convention, and on further simplification, we obtain

$$
\mathscr{L}_{2}\left[\int_{a}^{x} \int_{a}^{y} f(t, \tau) \Delta \tau \Delta t\right]=\frac{\widetilde{\widetilde{F}}(p, q)}{p q} .
$$

\section{Example 4}

(a) Solve the partial difference equation:

$\Delta_{x} u(x, y)-\Delta_{y} u(x, y)=0$

with $u(x, a)=(x-a)^{\frac{1}{1}}, u(a, y)=(y-a)^{\frac{1}{1}}$.

Application of the delta Laplace transforms to initial conditions by Lemma 3,

$$
\begin{aligned}
& \mathscr{L}_{x} u(x, a)=\mathscr{L}_{x}(x-a)^{\underline{1}}=\frac{1}{p^{2}}, \\
& \mathscr{L}_{y} u(a, y)=\mathscr{L}_{y}(y-a)^{\underline{1}}=\frac{1}{q^{2}} .
\end{aligned}
$$

Apply the delta double Laplace transforms to difference equation and then use linearity property:

$$
\begin{aligned}
\mathscr{L}_{2}\left[\Delta_{x} u(x, y)-\Delta_{y} u(x, y)\right] & =0, \\
\mathscr{L}_{2} \Delta_{x} u(x, y)-\mathscr{L}_{2} \Delta_{y} u(x, y) & =0 .
\end{aligned}
$$

Using Theorem 9,

$$
\begin{aligned}
& \quad\left[p \mathscr{L}_{2}\{u(x, y)\}-\mathscr{L}_{y}\{u(a, y)\}\right] \\
& \quad-\left[q \mathscr{L}_{2}\{u(x, y)\}-\mathscr{L}_{x}\{u(x, a)\}\right]=0, \\
& (p-q) \mathscr{L}_{2}\{u(x, y)\}=\frac{1}{q^{2}}-\frac{1}{p^{2}}, \\
& \mathscr{L}_{2}\{u(x, y)\}=\frac{1}{p q^{2}}+\frac{1}{p^{2} q} .
\end{aligned}
$$

Inverting the delta Laplace transforms pairs

$$
u(x, y)=(x-a)^{\underline{1}}+(y-a)^{\underline{1}} .
$$

(b) Solve the same partial difference equation with slightly different initial conditions: 


$$
\begin{aligned}
& u(x, a)=(x-a)^{2} \\
& u(a, y)=(y-a)^{2}
\end{aligned}
$$

Application of the delta Laplace transforms to initial conditions by Lemma 3:

$$
\begin{aligned}
& \mathscr{L}_{x} u(x, a)=\mathscr{L}_{x}(x-a)^{\frac{2}{}}=\frac{2}{p^{3}}, \\
& \mathscr{L}_{y} u(a, y)=\mathscr{L}_{y}(y-a)^{\frac{2}{2}}=\frac{2}{q^{3}} .
\end{aligned}
$$

From equation $(7)(p-q) \mathscr{L}_{2}\{u(x, y)\}=\frac{2}{q^{3}}-\frac{2}{p^{3}}$,

$$
\mathscr{L}_{2}\{u(x, y)\}=\frac{2}{p q^{3}}+\frac{2}{p^{2} q^{2}}+\frac{2}{p^{3} q} .
$$

Inverting delta Laplace transforms pairs,

$$
u(x, y)=(x-a)^{\frac{2}{2}}+2(x-a)^{\frac{1}{1}}(y-a)^{\frac{1}{}}+(y-a)^{\frac{2}{2}} .
$$

Assume $f: \mathbb{N}_{a} \longrightarrow \mathbb{R}$, then the Riemann-Liouville fractional difference of order $N-1<\alpha \leq N$, for $N \in \mathbb{N}_{1}$ is given by $\Delta_{a}^{\alpha} f(x)=\Delta^{N} \Delta_{a}^{-(N-\alpha)} f(x)$, for $x \in \mathbb{N}_{a+N-\alpha}$. By using the discussion and results, from Theorem 2.65 to Theorem 2.70, in [10], we take the starting point of the double Laplace $a+\alpha-N$ and $a+N-\alpha$, respectively, for sum and difference operator.

Corollary 2. Assume $u(x, y): \mathbb{N}_{a} \times \mathbb{N}_{a} \longrightarrow \mathbb{R}$, such that the delta double Laplace transforms exists for constants $p \neq-1$ and $q \neq-1$ and denote $\mathscr{L}_{2} u(x, y)=\tilde{\widetilde{u}}(p, q)$. Then, for $\lceil N-\alpha\rceil=M$ and $\lceil k+\alpha-N\rceil=L$, the delta double Laplace transforms of fractional order operators is given by

(i) $\mathscr{L}_{2}\left[\Delta_{a}^{-\alpha} u(x, y)\right](p, q)=\frac{(p+1)^{\alpha-N}(q+1)^{\alpha-N}}{p^{\alpha} q^{\alpha}} \tilde{\tilde{u}}(p, q), \quad$ where $N-1<\alpha<N$,

(ii) $\mathscr{L}_{2}\left[\Delta_{x}^{\alpha} u(x, y)\right](p, q)=p^{\alpha} q^{\alpha-N}(p+1)^{N-\alpha-M}(q+1)^{N-\alpha-M} \widetilde{\widetilde{u}}(p, q)$

$$
-\sum_{k=0}^{N-1} p^{N-1-k}\left[q^{k+\alpha-N}(q+1)^{L-(k+\alpha-N)} \widetilde{u}(a, q)-\sum_{j=0}^{L-1} q^{j} \Delta_{x}^{k+\alpha-N-1} u(a, a+L-(k+\alpha-N))\right],
$$

$$
\text { (iii) } \begin{aligned}
\mathscr{L}_{2}\left[\Delta_{y}^{\alpha} u(x, y)\right](p, q)= & p^{\alpha-N} q^{\alpha}(p+1)^{N-\alpha-M}(q+1)^{N-\alpha-M} \widetilde{\widetilde{u}}(p, q) \\
& -\sum_{k=0}^{N-1} q^{N-1-k}\left[p^{k+\alpha-N}(p+1)^{L-(k+\alpha-N)} \widetilde{u}(p, a)-\sum_{j=0}^{L-1} p^{j} \Delta_{y}^{k+\alpha-N-1} u(a+L-(k+\alpha-N), a)\right] .
\end{aligned}
$$

Proof

(i) Proof is an implication of Definition 3.1 and Theorem 2.67 of [10]

(ii) Result is obtained by application of Theorem 5.4 part (i) and Theorem 2.70 of [10]

(iii) Result is obtained by application of Theorem 5.4 part (ii) and Theorem 2.70 of [10]

Example 5. Solve the fractional difference equation for $0<\alpha<1$ :

$$
\Delta_{x}^{\alpha} u(x, y)=(y-a)^{\frac{1}{}}, \quad \text { with } u(a, y)=0 .
$$

Apply the delta Laplace transforms to initial condition $\mathscr{L}_{y} u(a, y)=\mathscr{L}_{y} 0=0$. For $0<\alpha<1$, we have $N=1$ which implies $\quad k=0$ and therefore $\lceil k+\alpha-1\rceil=L=0$, also $\lceil 1-\alpha\rceil=M=1$. Application of the delta double Laplace transforms on both sides of fractional difference equation (103) and making use of equation (101) on left- hand side, and on the right-hand side we used Example 3 to obtain

$$
\frac{p^{\alpha} q^{\alpha-1}}{(p+1)^{\alpha}(q+1)^{\alpha}} \tilde{\widetilde{u}}(p, q)-p^{0} q^{\alpha-1}(q+1)^{1-\alpha} \widetilde{u}(a, q)=\frac{1}{p q^{2}} .
$$

Using $\widetilde{u}(a, q)=0$ and simplifying the above,

$$
\widetilde{\tilde{u}}(p, q)=\frac{(p+1)^{\alpha}(q+1)^{\alpha}}{p^{\alpha+1} q^{\alpha+1}} \text {. }
$$

Inverting the delta Laplace transforms pairs by making use of Theorem 1 (iii), together with Lemma 3 (iii),

$$
u(x, y)=\frac{(x-a)^{\underline{\alpha}}}{\Gamma(\alpha+1)} \frac{(y-a)^{\underline{\alpha}}}{\Gamma(\alpha+1)} \text {. }
$$

\section{Data Availability}

The data used to support the findings of this study are available from the corresponding author upon request. 


\section{Conflicts of Interest}

The authors declare no conflicts of interest.

\section{Authors' Contributions}

All authors contributed in writing review and editing the article and have read and agreed to the published version of the manuscript.

\section{Acknowledgments}

The authors would like to thank Prince Sultan University for funding this work through research group Nonlinear Analysis Methods in Applied Mathematics (NAMAM), Group no. RG-DES-2017-01-17.

\section{References}

[1] C. Jordan, Calculus of Finite Differences, AMS Chelsea Publishing, Chelsea, NY, USA, 1979.

[2] M. Bohner and A. C. Peterson, Dynamic Equations on Time Scales an Introduction with Applications, Birkhauser Boston, Cambridge, MA, USA, 2001.

[3] T. Abdeljawad and D. Baleanu, "On fractional derivatives with exponential kernel and their discrete versions," Reports on Mathematical Physics, vol. 80, no. 1, pp. 11-27, 2017.

[4] T. Abdeljawad, "On Riemann and Caputo fractional differences," Computers \& Mathematics with Applications, vol. 62, no. 3, pp. 1602-1611, 2011.

[5] T. Abdeljawad, D. Baleanu, F. Jarad, and R. P. Agarwal, "Fractional sums and differences with binomial coefficients," Discrete Dynamics in Nature and Society, vol. 2013, Article ID 104173, 6 pages, 2013.

[6] T. Abdeljawad, S. Banerjee, and G. C. Wu, "Discrete tempered fractional calculus for new chaotic systems with short memory and image encryption," Optik, Article ID 163698, 2019.

[7] T. Abdeljawad, "On delta and nabla Caputo fractional differences and dual identities," Discrete Dynamics in Nature and Society, vol. 2013, Article ID 406910, 12 pages, 2013.

[8] F. M. Atici and P. W. Eloe, "A transform method in discrete fractional calculus," International Journal of Differential Equations, vol. 2, no. 2, 2007.

[9] A. Ivan, J. Losada, and J. J. Nieto, "On quasi-periodic properties of fractional sums and fractional differences of periodic functions," Applied Mathematics and Computation, vol. 273, pp. 190-200, 2016.

[10] C. S. Goodrich and A. C. Peterson, Discrete Fractional Calculus, Springer, New York, NY, USA, 2015.

[11] C. S. Goodrich, "Existence and uniqueness of solutions to a fractional difference equation with nonlocal conditions," Computers \& Mathematics with Applications, vol. 61, no. 2, pp. 191-202, 2011.

[12] L.-L. Huang, J. H. Park, G.-C. Wu, and Z.-W. Mo, "Variableorder fractional discrete-time recurrent neural networks," Journal of Computational and Applied Mathematics, vol. 370, p. 112633, 2020.

[13] G.-C. Wu, D. Baleanu, and W.-H. Luo, "Lyapunov functions for Riemann-Liouville-like fractional difference equations," Applied Mathematics and Computation, vol. 314, pp. 228-236, 2017.
[14] G. C. Wu, Z. G. Deng, D. Baleanu, and D. Q. Zeng, "New variable-order fractional chaotic systems for fast image encryption," Chaos, vol. 29, Article ID 083103, 2019.

[15] M. T. Holm, "The Laplace transform in discrete fractional calculus," Computers \& Mathematics with Applications, vol. 62, no. 3, pp. 1591-1601, 2011.

[16] M. T. Holm, The Theory of Discrete Fractional Calculus: Development and Application, University of Nebraska-Lincoln, Lincoln, NE, USA, 2011.

[17] S. S. Haider and M. U. Rehman, "Ulam-Hyers-Rassias stability and existence of solutions to nonlinear fractional difference equations with multipoint summation boundary condition," Acta Mathematica Scientia, vol. 40, no. 2, pp. 589-602, 2020.

[18] S. S. Haider and M. U. Rehman, "Construction of fixed point operators for nonlinear difference equations of non integer order with impulses," Fractional Calculus and Applied Analysis, vol. 23, no. 3, 2020.

[19] F. Jarad, K. Taş, and K. Ta, "A new transform method in nabla discrete fractional calculus," Advances in Difference Equations, vol. 2012, no. 1, 190 pages, 2012.

[20] D. L. Bernstein, “The double Laplace integral," Duke Mathematical Journal, vol. 8, no. 3, pp. 460-496, 1941.

[21] G. A. Coon and D. L. Bernstein, Some Properties of the Double Laplace Transformation, Taylor Instrument Companies, University of Rochester Press, Rochester, NY, USA, 1953.

[22] G. A. Coon and D. L. Bernstein, "Some general formulas for double Laplace transformations," Proceedings of the American Mathematical Society, vol. 14, no. 1, p. 52, 1963.

[23] L. Debnath, "The double Laplace transforms and their properties with applications to functional, integral and partial differential equations," International Journal of Applied and Computational Mathematics, vol. 2, no. 2, pp. 223-241, 2016.

[24] R. R. Dhunde and G. L. Waghmare, "On some convergence theorems of double Laplace transform," Journal of Informatics and Mathematical Sciences, vol. 6, pp. 45-54, 2014.

[25] A. Atangana, "A note on the triple Laplace transform and its applications to some kind of third-order differential equation," Abstract and Applied Analysis, vol. 2013, Article ID 769102, 10 pages, 2013.

[26] R. S. Dahiya and M. Vinayagamoorthy, "Laplace transform pairs of n-dimensions and heat conduction problem," Mathematical and Computer Modelling, vol. 13, no. 10, pp. 35-50, 1990.

[27] H. U. Rehman, M. Iftikhar, S. Saleem, M. Younis, and A. Mueed, "A computational quadruple Laplace transform for the solution of partial differential equations," Applied Mathematics, vol. 5, no. 21, pp. 3372-3382, 2014.

[28] S. S. Haider, M. U. Rehman, and T. Abdeljawad, "On Hilfer fractional difference operator," Advances in Difference Equations, vol. 122, pp. 1-20, 2020.

[29] S. S. Haider and M. U. Rehman, "On substantial fractional difference operator," Advances in Difference Equations, no. 1, , 2020.

[30] M. Bohner, G. S. Guseinov, and B. Karpuz, "Further properties of the Laplace transform on time scales with arbitrary graininess," Integral Transforms and Special Functions, vol. 24, no. 4, pp. 289-301, 2013.

[31] M. D. Ortigueira, F. J. V. Coito, and J. J. Trujillo, "Discretetime differential systems," Signal Processing, vol. 107, pp. 198-217, 2015.

[32] P. Savoye, "On the inclusion of difference equation problems and Z Transform methods in sophomore differential equation classes," Primus, vol. 19, no. 6, pp. 491-499, 2009. 
[33] M. Omran and A. Kiliman, "Fractional double Laplace transform and its properties," in Proceedings of the AIP Conference, vol. 1795, no. 1, January 2017.

[34] J. Peng and K. Li, "A note on property of the Mittag-Leffler function," Journal of Mathematical Analysis and Applications, vol. 370, no. 2, pp. 635-638, 2010.

[35] B. Zhang and Y. Zhou, Qualitative Analysis of Delay Partial Difference Equations, Contemporary Mathematics and its Applications, Hindawi, New York, NY, USA, 2007.

[36] F. Ozpinar and F. M. Belgacem, "The discrete homotopy perturbation Sumudu transform method for solving partial difference equations," Discrete \& Continuous Dynamical Systems, vol. 12, no. 3, pp. 615-624, 2019.

[37] A. Aghili and M. R. Masomi, "Solving systems of fractional partial differential equations via two dimensional Laplace transforms," International Journal of ResearchGranthaalayah, vol. 5, no. 12, pp. 406-420, 2017.

[38] S. K. Elagan, M. Sayed, and M. Higazy, "An analytical study on fractional partial differential equations by Laplace transform operator method," International Journal of Applied Engineering Research, vol. 13, no. 1, pp. 545-549, 2018.

[39] A. M. Haghighi and D. P. Mishev, Difference and Differential Equations with Applications in Queueing Theory, John Wiley and Sons, Hoboken, NJ, USA, 2013.

[40] A. M. O. Anwar, F. Jarad, D. Baleaun, and F. Ayaz, "Fractional Caputo heat equation within the double Laplace transform," Romanian Journal of Physics, vol. 58, pp. 15-18, 2013. 